\title{
Modeling of Intensity-Modulated Photocurrent/Photovoltage Spectroscopy: Effect of Mobile Ions on the Dynamic Response of Perovskite Solar Cells
}

\author{
David Bernhardsgrütter*(i) and Matthias M. Schmid \\ Institute of Computational Physics, Zurich University of Applied Sciences, Wildbachstr. 21, 8401 Winterthur, Switzerland
}

\begin{abstract}
Intensity-modulated photocurrent spectroscopy (IMPS) and intensitymodulated photovoltage spectroscopy (IMVS) have proven to provide insights into the charge carrier dynamics of perovskite solar cells (PSCs), though the interpretation of measured spectra is not straightforward. Using a one-dimensional drift-diffusion model, we investigate the effect of mobile ions on the small signal response of PSCs. The IMPS and IMVS response is derived by sinusoidal steady-state analysis and Fourier decomposition (FD) of the transient cell response to a light intensity step. The FD method links the time and frequency domain, giving an additional perspective for the interpretation of the results at the expense of numerical efficiency.

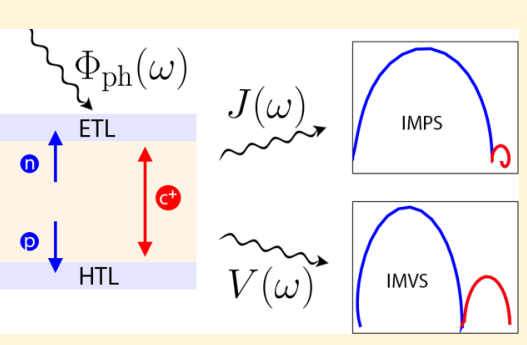
The simulated spectra are characterized by two separate arcs, where the high-frequency response is attributed to the transport and recombination of electronic charges, while the low-frequency peak represents the transport of ions. We show that this model is able to reproduce qualitatively the measurements reported in the literature. The concepts presented in this paper are applicable for the analysis of the small signal response of any mixed ionic electronic conductor.
\end{abstract}

\section{INTRODUCTION}

Since their advent in 2009, halide perovskite solar cells (PSCs) underwent a rapid development in terms of efficiency. ${ }^{1,2}$ Furthermore, their potential for low manufacturing costs, the ideal absorption properties, and high open-circuit voltage make them a promising candidate to be combined as a top cell with a bottom silicon cell to form a tandem solar cell.

Numerical simulation of PSCs based on mathematical models describing absorption, charge transport, and recombination of electrons, holes, and ions can contribute significantly to further understand the physical processes in the cell. Usually, the PSCs are modeled by coupling semiconductor equations describing transport of electrons and holes (by drift-diffusion) equipped with a recombination term to equations for the ionic movement. Taking ion transport into account, the model has given further evidence for compatibility of hysteresis and mobile ions. ${ }^{3-7}$ The primary effect of the mobile ions is screening of the internal electric field. A plethora of slow transient effects exhibited by PSCs could be explained and reproduced with this model. ${ }^{8-10}$ Owing to the mixed ionic and electronic nature of charge transport, the models used in the perovskite community are akin to the ones presented in papers discussing light-emitting electrochemical cells (LECs) and mixed ionic electronic conductors (MIECs). ${ }^{11,12}$

In refs 4 and 9, the drift-diffusion model is solved in two steps. First, an asymptotic method is used to calculate the electric potential caused by the mobile ions. Then, in the second step, the drift-diffusion calculation for the electrons and holes is performed. In ref 10, the electronic-ionic system was fully computed without the simplification of the asymptotic method ${ }^{4}$ or fixed accumulation layers. ${ }^{6}$ References 4 and 10 have in common that their modeling domain is restricted to the perovskite layer, such that the electron transport layer (ETL) and hole transport layer (HTL) are treated as metal-semiconductor interfaces with a defined barrier height and surface recombination velocities. References 3, 5, 6, 13 take ETL and HTL layers explicitly into account by solving the drift-diffusion model for electrons and holes in the ETL/HTL as well.

An important class of characterization techniques consists in applying small perturbations to the cell's stationary state and analyzing its response. Examples of this kind of characterization techniques are impedance spectroscopy (IS), intensitymodulated photocurrent spectroscopy (IMPS), and intensitymodulated photovoltage spectroscopy (IMVS). Intensitymodulated spectroscopy differs from IS by the type of perturbation: while the cell is excited by a small electrical voltage for IS, the cell is exposed to a small sinusoidal monochromatic illumination for IMPS and IMVS. In all cases, one measures the cell response either in current (IS and IMPS) or voltage (IMVS).

Small-perturbation techniques have been applied to elucidate transport and recombination mechanisms in PSCs. ${ }^{14,15}$ Yet a comprehensive theory for the interpretation of the IMPS and IMVS response of PSCs is still lacking. In this work, we demonstrate how numerical simulations facilitate the identification of the physical processes underlying each arc of the spectra, without regressing to an equivalent circuit model as discussed in ref 16 .

Received: September 5, 2019

Revised: November 22, 2019

Published: November 22, 2019 
The present paper is organized as follows: In Section 2, we describe our modeling approach in detail. In particular, we explain how the transfer functions of IMPS and IMVS can consistently be calculated directly from the model equations. This is done using two standard methods [sinusoidal steadystate analysis $\left(\mathrm{S}^{3} \mathrm{~A}\right)$ and Fourier decomposition (FD)] for the IS of semiconductor devices, which we adopt for IMPS and IMVS. The simulations are then verified by comparing the two methods. In Section 3, we apply our model to investigate the influence of ion transport on IMPS and IMVS measurements. We perform several parameter studies to reveal that the highfrequency response is linked to transport and recombination of electronic charges, while the low-frequency response of PSCs is associated with mobile ions. Finally, in the last section, we summarize our results.

\section{METHODS}

We consider a PSC with planar device structure. A perovskite film of thickness $d$ is sandwiched between an n-type ETL and a p-type HTL. The interfaces are located at $x=0$ (ETL) and $x=$ $d$ (HTL). This compound forms an $\mathrm{n}-\mathrm{i}-\mathrm{p}$ junction, which is sealed by a transparent conductive oxide and a metal contact.

We assume that the potential is approximately constant across the ETL and HTL. This simplification is justified by highly doped or, more generally, highly conductive adjacent layers. The modeling domain is thus reduced to the perovskite film $x \in[0, d]$. Peripheral losses are modeled by considering the $R C$ time constant in the derivation of the IMPS transfer function.

2.1. Bulk Equations. The perovskite material is treated as a homogeneous medium which conducts both electronic charge carriers and ions (MIEC). The continuity equation of species $i=n, p, a$, and $c$ (referring to electrons, holes, anions, and cations, respectively) is given by

$$
\frac{\partial c_{i}}{\partial t}+\frac{1}{z_{i} q} \frac{\partial J_{i}}{\partial x}=G_{i}-R_{i}
$$

Here, $c_{i}, J_{i}$, and $z_{i}$ are the particle density, current density, and charge number of species $i$, respectively, and $q$ is the elementary charge. We assume that ions are conserved during cell operation, $G_{i}=R_{i}=0$ for $i=a, c$. A Lambert-Beer model is used for the generation term of electrons and holes. As intensity-modulated techniques are typically measured with monochromatic illumination, the generation term reads $G_{n}=$ $G_{p}=\alpha \Phi_{\mathrm{ph}} \exp (-\alpha x)$, where $\alpha$ is the absorption coefficient and $\Phi_{\mathrm{ph}}$ is the incident photon flux. We assume that trap-assisted recombination is the dominant recombination mechanism in the bulk. It is modeled by the Shockley-Reed-Hall (SRH) expression (given here for midgap traps).

$$
R_{n}=R_{p}=\frac{n p-n_{i}^{2}}{\tau_{p}\left(n+n_{i}\right)+\tau_{n}\left(p+n_{i}\right)}
$$

where $\tau_{n}$ and $\tau_{p}$ are the SRH lifetimes of electrons and holes and $n_{i}$ is the intrinsic carrier density.

Carrier transport by drift and diffusion is considered

$$
J_{i}=-z_{i} q D_{i} \frac{\partial c_{i}}{\partial x}-q c_{i} \mu_{i} \frac{\partial \Phi}{\partial x}
$$

where $\Phi$ is the electrostatic potential. We assume Einstein's relation for the diffusion coefficient $D_{i}$ and mobility $\mu_{i}$. The model is completed by Poisson's equation of electrostatics.

$$
\frac{\partial^{2} \Phi}{\partial x^{2}}=-\frac{q}{\varepsilon_{\mathrm{r}} \varepsilon_{0}} \sum_{i} z_{i} c_{i}
$$

where $\varepsilon_{0}$ and $\varepsilon_{\mathrm{r}}$ are the permittivities of free space and the relative dielectric constant, respectively.

The total current $J$ is composed of the particle currents $J_{i}$ and Maxwell's displacement current

$$
J=\sum_{i} J_{i}+\varepsilon_{\mathrm{r}} \varepsilon_{0} \frac{\partial E}{\partial t}
$$

where $E=-\partial \Phi / \partial x$ is the electric field.

2.2. Initial and Boundary Conditions. We assume that the adjacent charge-collecting layers are ionically insulating

$$
J_{i}(0)=J_{i}(d)=0, \quad i=a, c
$$

The total amount of mobile ions in the domain is thus conserved

$$
\frac{1}{d} \int_{0}^{d} c_{a} \mathrm{~d} x=\frac{1}{d} \int_{0}^{d} c_{c} \mathrm{~d} x=c_{0}
$$

Schottky-type boundary conditions are imposed at the contacts

$$
\begin{aligned}
& J_{i}(0)=-z_{i} q v_{i}^{0}\left(c_{i}(0)-\bar{c}_{i}^{0}\right) \text { And } \\
& J_{i}(d)=z_{i} q v_{i}^{d}\left(c_{i}(d)-\bar{c}_{i}^{d}\right), \quad i=n, p
\end{aligned}
$$

Here, $v_{i}$ is the surface recombination velocity (SRV) and $\bar{c}_{i}$ refers to the equilibrium carrier density of species $i$, superscripts indicate the respective contacts. The SRV $v_{i}$ describes recombination through interface states, which reflects the chemical passivation of the interface. In the limiting case of $v_{n}^{0} \rightarrow \infty$ and $v_{p}^{0}=0$ at the ETL contact, the Robin-type boundary condition 8 transitions into mixed Dirichlet and Neumann boundary conditions often found in models of PSCs, ${ }^{4,8}$ postulating perfectly selective contacts and sufficient extraction of the majority carriers. ${ }^{17}$

The boundary condition 8 is usually applied to semiconductor-metal contacts. As we assume high doping of the adjacent layers, their Fermi levels $E_{\mathrm{F}_{n}}^{\mathrm{ETL}}$ and $E_{\mathrm{F}_{p}}^{\mathrm{HTL}}$ take the role of the metal work functions. The difference of the Fermi levels in the charge-collecting layers raise a built-in voltage $V_{\mathrm{bi}}=E_{\mathrm{F}_{n}}^{\mathrm{ETL}}-E_{\mathrm{F}_{p}}^{\mathrm{HTL}}$. The built-in voltage can be rewritten as

$$
V_{\mathrm{bi}}=E_{\mathrm{g}}-\Phi_{\mathrm{B}}^{0}-\Phi_{\mathrm{B}}^{d}
$$

where $E_{\mathrm{g}}$ is the band gap of the perovskite material and $\Phi_{\mathrm{B}}$ refers to the injection barrier at the contact. The barrier heights are given by $\Phi_{\mathrm{B}}^{0}=E_{\mathrm{C}}-E_{\mathrm{F}_{n}}^{\mathrm{ETL}}$ and $\Phi_{\mathrm{B}}^{d}=E_{\mathrm{F}_{p}}^{\mathrm{HTL}}-E_{\mathrm{V}}$, where $E_{\mathrm{C}}$ and $E_{\mathrm{V}}$ are the perovskite conduction and valence bands. The equilibrium carrier densities are then related to the injection barrier by

$$
\bar{c}_{n}=N_{\mathrm{C}} \exp \left(-\frac{q \Phi_{\mathrm{B}}}{k_{\mathrm{B}} T}\right), \bar{c}_{p}=N_{\mathrm{V}} \exp \left(-\frac{q\left(E_{\mathrm{g}}-\Phi_{\mathrm{B}}\right)}{k_{\mathrm{B}} T}\right)
$$

Here, $N_{\mathrm{C}}$ and $N_{\mathrm{V}}$ are the effective density of states (DOS) of the valence and conduction bands, respectively, $k_{\mathrm{B}}$ is the Boltzmann constant, and $T$ the temperature.

For an externally applied voltage $V_{\text {app}}$, the potential drop across the perovskite layer is given by $V=V_{\mathrm{app}}-V_{\mathrm{bi}}$. The potential at the interface to the ETL is defined as zero, which 
results in the following set of boundary conditions for the potential

$$
\Phi(0)=0, \quad \Phi(d)=V_{\text {app }}-V_{\mathrm{bi}}
$$

Open-circuit conditions are modeled by introducing the additional boundary condition $J_{\text {app }}=0$, while $V_{\text {app }}$ is treated as a variable and the additional ordinary differential equation $V_{\text {app }}^{\prime}$ $=J\left(V_{\text {app }}\right)-J_{\text {app }}$ is solved with an initial voltage $V_{\text {init }}$ close to $V_{\text {OC }}$. For the transient simulations, we use the initial conditions consistent with the stationary solution.

2.3. Intensity-Modulated Photocurrent/Photovoltage Spectroscopy. A small sinusoidal perturbation with angular frequency $\omega=2 \pi f$ is superimposed on a steady illumination, $\Phi_{\mathrm{ph}}(t)=\Phi_{\mathrm{ph}}+\delta \Phi_{\mathrm{ph}} \exp (i \omega t)$ with $\delta \ll 1$. The current response $J(t)=\hat{J}+\tilde{J} \exp (i \omega t)$ for a cell kept at short circuit consists of a large stationary current $\hat{J}$ and a small perturbed component $\tilde{J}$. The internal IMPS transfer function is then defined as

$$
Z_{\mathrm{IMPS}}^{\mathrm{int}}=\frac{\tilde{J}}{q \delta \Phi_{\mathrm{ph}}}
$$

The measured IMPS signal deviates from the idealized IMPS response by $R C$ attenuation, which is particularly distinct at high frequencies. For an electrode capacitance $C$ and resistance $R$, the attenuation factor is given by ${ }^{18}$

$$
A(\omega)=\frac{1}{1+i \omega R C}
$$

The external IMPS transfer function is then given by

$$
Z_{\mathrm{IMPS}}^{\mathrm{ext}}=A(\omega) Z_{\mathrm{IMPS}}^{\mathrm{int}}
$$

Analogously, the voltage response $V=\hat{V}+\tilde{V} \exp (i \omega t)$ for a cell kept at open circuit comprises a stationary voltage $\hat{V}$ and a perturbation $\tilde{V}$. The IMVS transfer function is given by

$$
Z_{\mathrm{IMVS}}=\frac{\tilde{V}}{q \delta \Phi_{\mathrm{ph}}}
$$

In the following sections, we discuss two different strategies to compute $Z_{\text {IMPS }}$ and $Z_{\text {IMvs }}$.

2.4. Sinusoidal Steady-State Analysis $\left(S^{3} A\right)$. The $S^{3} A$ method has usually been applied for the numerical analysis of the admittance of semiconductor devices. ${ }^{19}$ The concept is easily transferable to the IMPS and IMVS case. The bulk equations are linearized around the stationary operating point $\hat{c}_{i}$, $\hat{\Phi}$. By substituting $c_{i}=\hat{c}_{i}+\tilde{c}_{i} \exp (i \omega t)$ and $\Phi=\tilde{\Phi}+\tilde{\Phi}$ $\exp (i \omega t)$ in eqs $1-4$ and calculating the first-order Taylor expansion (the linearity assumption), one obtains a timeindependent linear system for the perturbed components $\tilde{c}_{i}, \tilde{\Phi}$, which is solved for each frequency. The boundary conditions for the perturbed system are obtained analogously.

2.5. Fourier Decomposition of Transient Excitation (FD). We monitor the cell response to a small light intensity step at $t=0$, where the cell is in steady state at $t=0^{-}$and is constantly held at either short circuit (IMPS) or open circuit (IMVS). The time-dependent illumination intensity is given by $\Phi_{\mathrm{ph}}(t)=\Phi_{\mathrm{ph}}+\delta \Phi_{\mathrm{ph}} H(t)$, where $H(t)$ is the Heaviside step function and $\delta \ll 1$. The transfer functions can then be written as Fourier transform of the transient excitation

$$
\begin{aligned}
Z_{\mathrm{IMPS}} & =\frac{\mathcal{F}\left\{J(t)-J\left(0^{-}\right)\right\}}{\mathcal{F}\left\{q \Phi_{\mathrm{ph}}(t)-q \Phi_{\mathrm{ph}}\left(0^{-}\right)\right\}}, \\
Z_{\mathrm{IMVS}} & =\frac{\mathcal{F}\left\{V(t)-V\left(0^{-}\right)\right\}}{\mathcal{F}\left\{q \Phi_{\mathrm{ph}}(t)-q \Phi_{\mathrm{ph}}\left(0^{-}\right)\right\}}
\end{aligned}
$$

The Fourier transform of the Heaviside function evaluates to $\mathcal{F}\left\{q \Phi_{\mathrm{ph}}(t)-q \Phi_{\mathrm{ph}}\left(0^{-}\right)\right\}=q \delta \Phi_{\mathrm{ph}} / i \omega$. We separate the Fourier transform of the cell response into real and imaginary parts to obtain the following expressions for the transfer functions.

$$
\begin{aligned}
\operatorname{Re}\left(Z_{\mathrm{IMPS}}\right)= & \frac{J(\infty)-J\left(0^{-}\right)}{q \delta \Phi_{\mathrm{ph}}} \\
& +\frac{\omega}{q \delta \Phi_{\mathrm{ph}}} \int_{0}^{\infty}(J(t)-J(\infty)) \sin (\omega t) \mathrm{d} t \\
\operatorname{Im}\left(Z_{\mathrm{IMPS}}\right)= & \frac{1}{q \delta \Phi_{\mathrm{ph}}} \int_{0}^{\infty}(J(t)-J(\infty)) \cos (\omega t) \mathrm{d} t \\
\operatorname{Re}\left(Z_{\mathrm{IMVS}}\right)= & \frac{V(\infty)-V\left(0^{-}\right)}{q \delta \Phi_{\mathrm{ph}}} \\
& +\frac{\omega}{q \delta \Phi_{\mathrm{ph}}} \int_{0}^{\infty}(V(t)-V(\infty)) \sin (\omega t) \mathrm{d} t \\
\operatorname{Im}\left(Z_{\mathrm{IMVS}}\right)= & \frac{1}{q \delta \Phi_{\mathrm{ph}}} \int_{0}^{\infty}(V(t)-V(\infty)) \cos (\omega t) \mathrm{d} t
\end{aligned}
$$

\begin{tabular}{|c|c|c|c|}
\hline description, reference & symbol & value & unit \\
\hline perovskite layer thickness & $d$ & 500 & $\mathrm{~nm}$ \\
\hline relative permittivity ${ }^{25}$ & $\varepsilon_{\mathrm{r}}$ & 62 & \\
\hline conduction band minimum ${ }^{a}$ & $E_{\mathrm{C}}$ & -4.3 & $\mathrm{eV}$ \\
\hline valence band maximum & $E_{\mathrm{V}}$ & -5.9 & $\mathrm{eV}$ \\
\hline effective DOS conduction band ${ }^{26}$ & $N_{\mathrm{C}}$ & $6.98 \times 10^{18}$ & $\mathrm{~cm}^{-3}$ \\
\hline effective DOS valence band ${ }^{26}$ & $N_{\mathrm{V}}$ & $2.49 \times 10^{18}$ & $\mathrm{~cm}^{-3}$ \\
\hline ionic density at equilibrium ${ }^{22,27}$ & $c_{0}$ & $1.6 \times 10^{19}$ & $\mathrm{~cm}^{-3}$ \\
\hline mobility electrons/holes ${ }^{b}$ & $\mu_{n}, \mu_{p}$ & 0.1 & $\mathrm{~cm}^{2} \mathrm{~V}^{-1} \mathrm{~s}^{-1}$ \\
\hline mobility cations ${ }^{c}$ & $\mu_{c}$ & $1 \times 10^{-10}$ & $\mathrm{~cm}^{2} \mathrm{~V}^{-1} \mathrm{~s}^{-1}$ \\
\hline mobility anions & $\mu_{a}$ & 0 & $\mathrm{~cm}^{2} \mathrm{~V}^{-1} \mathrm{~s}^{-1}$ \\
\hline electron/hole lifetime ${ }^{28,29}$ & $\tau_{n}, \tau_{p}$ & $5 \times 10^{-8}$ & $\mathrm{~s}$ \\
\hline barrier height & $\Phi_{\mathrm{B}}^{0}, \Phi_{\mathrm{B}}^{d}$ & 0.2 & $\mathrm{eV}$ \\
\hline SRV majority carrier & $v_{n}^{0}, v_{p}^{d}$ & $1 \times 10^{6}$ & $\mathrm{~cm} \mathrm{~s}^{-1}$ \\
\hline SRV minority carrier ${ }^{23}$ & $v_{p}^{0}, v_{n}^{d}$ & 450 & $\mathrm{~cm} \mathrm{~s}^{-1}$ \\
\hline $\begin{array}{l}\text { absorption coefficient at } \\
\lambda=625 \mathrm{~nm}^{30}\end{array}$ & $\alpha$ & 45000 & $\mathrm{~cm}^{-1}$ \\
\hline incident spectral photon flux ${ }^{d}$ & $\Phi_{\mathrm{ph}}$ & $3.15 \times 10^{17}$ & $\mathrm{~cm}^{-2} \mathrm{~s}^{-1}$ \\
\hline
\end{tabular}

Similar formulae have been derived for the admittance of semiconductor devices. ${ }^{19-21}$

2.6. Numerical Simulation. The list of model parameters is summarized in Table 1 . Following ref 4, we consider positively charged halide vacancies (cations) as mobile ionic species and therefore set the mobility of anions equal to zero. A recent paper (see ref 13, Supporting Information) suggests that including two types of mobile ionic species (one anionic and one cationic) in the model has a minor effect on the simulation results, if the mobility of the anions is much lower

Table 1. Standard Set of Parameters

${ }^{a}$ Corresponds to $E_{\mathrm{g}}=1.6 \mathrm{eV}$. Values are within the range reported in ref 31. ${ }^{b}$ Reported values range from 0.1 to $100 \mathrm{~cm}^{2} \mathrm{~V}^{-1} \mathrm{~s}^{-1}$, cf. refs 32 and 33. ${ }^{c}$ Reported values range from $10^{-10}$ to $10^{-6} \mathrm{~cm}^{2} \mathrm{~V}^{-1} \mathrm{~s}^{-1}$, cf. ref 34. ${ }^{d}$ Corresponds to $1000 \mathrm{~W} \mathrm{~m}^{-2}$ for monochromatic light with $\lambda=$ $625 \mathrm{~nm}$. 
than the mobility of the cations. The origin of ionic charge transport in PSCs is still under debate. ${ }^{2,23}$ The parameter values associated with mobile ions make the model numerically challenging. Ions accumulate in narrow boundary layers (the Debye length is given by $L_{\mathrm{D}}=\approx 2.3 \mathrm{~nm} \ll d$ ), and the time scales of ionic and electronic transport differ by several orders of magnitude $\left(\mu_{c} \ll \mu_{n}\right)$. Because of these unfavorable ratios, considerable effort has been put into the development of combined analytical and numerical methods, resulting in more benign numerical problems. ${ }^{4,8,9,24}$ Here, we opt for a purely numerical approach, and we will discuss the differences to methods adopted in the literature in the Results section.

The equations are spatially discretized by the ScharfetterGummel scheme in combination with nonuniform mesh refinement toward the edges. We use backward differentiation formula methods with exponentially distributed time steps for temporal discretization. The resulting nonlinear system of equations is solved with the Newton-Raphson method. All of the aforementioned methods are readily available in the commercial software tool Comsol Multiphysics 5.3a, which is used for all simulations. Similar methods have been successfully applied in the context of LECs or more generally for MIEC devices. ${ }^{11,12}$

In order to verify the simulation results, we compute the IMPS and IMVS response using both the $S^{3} A$ and FD techniques. The $\mathrm{S}^{3} \mathrm{~A}$ method is more efficient and accurate, as the system equations are formally linearized. ${ }^{19}$ For the FD method, we set $\delta=0.01$ as a compromise between numerical precision and linearity of the system response. A Levin-type integration method is used to numerically evaluate the highly oscillatory integrals.

The time steps of the transient simulation need to be chosen small $\left(\Delta t \ll \omega^{-1}\right)$ for the calculation of accurate highfrequency responses by means of FD. To reduce the computation time, we enforce the system to quickly reach steady state by increasing the mobility of the ions. Still, the response of the ionic and electronic subsystems is clearly distinguishable. We obtain an excellent agreement of the two methods, cf. Figures 1 and 2.

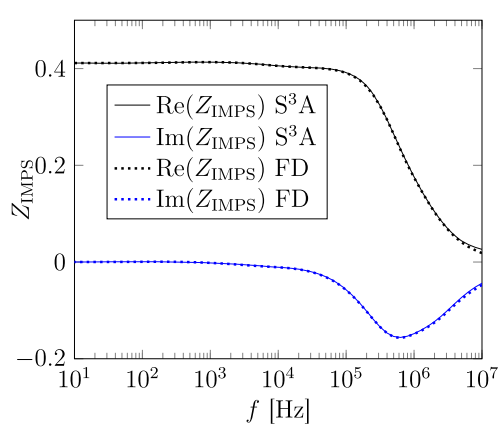

Figure 1. Real (black) and imaginary (blue) parts of the IMPS transfer function derived by the $\mathrm{S}^{3} \mathrm{~A}$ (solid line) and FD (dotted) methods. We set $\mu_{c}=1 \times 10^{-7} \mathrm{~cm}^{2} \mathrm{~V}^{-1} \mathrm{~s}^{-1}$ for this figure.

In the case of IMVS, we encounter difficulties to obtain convergence for the $\mathrm{S}^{3} \mathrm{~A}$ method. We therefore reduce the ionic density at equilibrium to verify both techniques. The FD method has proven to be numerically more robust. The IMVS spectra shown in the Results section are therefore derived with $\mathrm{FD}$, while we use $\mathrm{S}^{3} \mathrm{~A}$ to simulate the IMPS responses in the following.

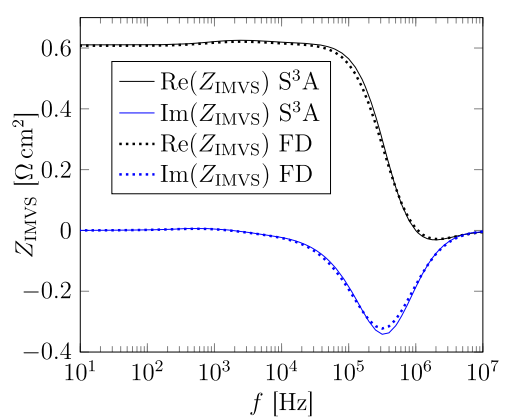

Figure 2. Real (black) and imaginary (blue) parts of the IMVS transfer function derived by the $\mathrm{S}^{3} \mathrm{~A}$ (solid line) and FD (dotted) methods. The parameters of the ionic subsystem are $c_{0}=1.6 \times 10^{17}$ $\mathrm{cm}^{-3}$ and $\mu_{c}=1 \times 10^{-5} \mathrm{~cm}^{2} \mathrm{~V}^{-1} \mathrm{~s}^{-1}$.

\section{RESULTS AND DISCUSSION}

Typical simulated IMPS and IMVS responses are illustrated in Figures 3 and 4, where two separate arcs are clearly distinguishable. The fast (high-frequency) component can be attributed to the transport and recombination of electronic charges, while the slow component (low-frequency) represents the transport of cations.

Similar low-frequency peaks of the IMPS and IMVS responses of PSCs have been reported in refs 8, 35-44 (IMPS) and refs 40-42, 45-47 (IMVS). The low-frequency response has been repeatedly attributed to ionic movement. Our simulation results qualitatively reproduce the reported measurements, further substantiating the hypothesis that the low-frequency arc is linked to the reorganization of slowly responding ions.

3.1. Influence of Ion Transport on the IMPS and IMVS Results. The influence of parameters associated with the ionic subsystem on the IMPS response is shown in Figure 3. Increasing the mobility of cations only causes a shift of the slow component to higher frequencies. Varying the ionic density involves a similar shift but also a more pronounced low-frequency response, as can be seen in Figure $3 \mathrm{~d}$. For high ion concentrations, $c_{c} \gg c_{n}$, the time constant extracted from the low-frequency peak matches with the dielectric relaxation time $\tau=\varepsilon_{\mathrm{r}} \varepsilon_{0} / q \mu_{c} c_{0}$. Notably, the low-frequency time constant is inversely proportional to both the mobility and the density of ions, $\tau_{\mathrm{lf}} \propto\left(\mu_{c} c_{0}\right)^{-1}$.

A shift of the slow component with the same qualitative trend as shown in Figure 3 has been reported in refs 35, 37, 38, where the ionic response has been linked with the average grain size of the perovskite layer. According to Figure 3, we can interpret this behavior as a dependence of the ion mobility or ionic density on the morphological structure of the perovskite film. This is in line with the analysis of ion migration in the context of film morphology, where a critical role has been assigned to grain boundaries. Faster ion transport at the grain boundaries than in grain interior has been reported. ${ }^{48-50}$

We observe two pronounced high-frequency arcs in the IMPS response for low ionic densities, cf. Figure 3d. Three distinct arcs have been reported, for example, in refs 14, 35, 37, 51. Therein, the high-frequency response has been attributed to the transport processes in the perovskite layer, while the intermediate arc has been attributed to the $\mathrm{TiO}_{2}$ scaffold. This reasoning is not applicable to our simulation results, as we consider solely the perovskite layer in our model. We performed additional simulations to determine the origin of 

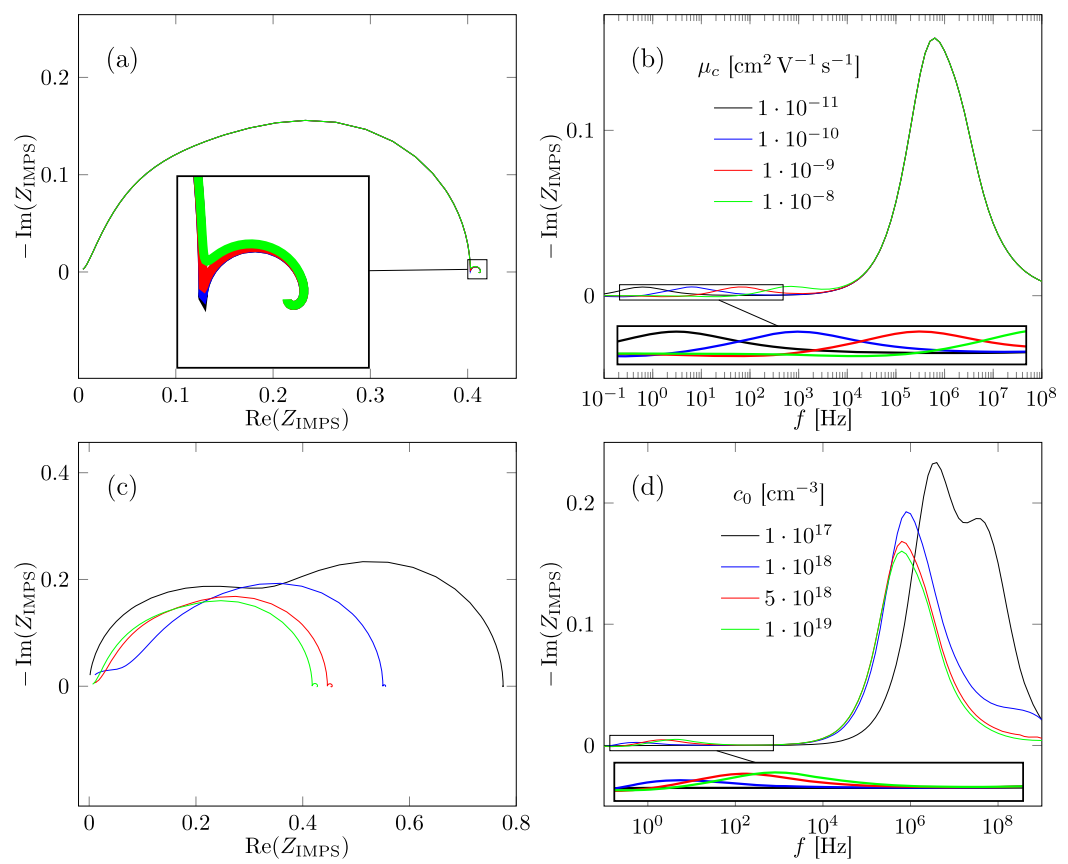

Figure 3. Simulated IMPS response for varying mobility $\mu_{c}(\mathrm{a}, \mathrm{b})$ and density $c_{0}(\mathrm{c}, \mathrm{d})$ of the mobile cations. Parts $(\mathrm{a}, \mathrm{c})$ show the Nyquist plot and parts $(b, d)$ show the imaginary part of the transfer function, revealing the effect of ionic mobility and density on the low-frequency peak.
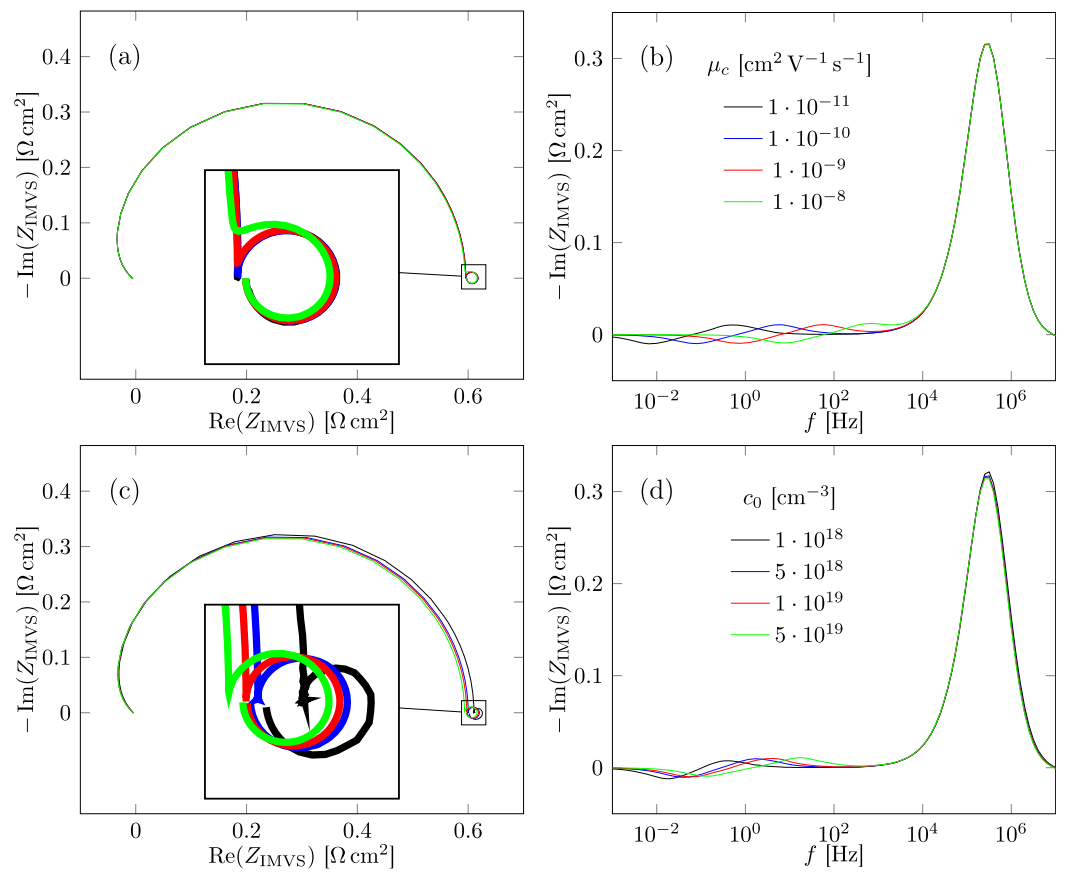

Figure 4. Effect of cation mobility $\mu_{c}(\mathrm{a}, \mathrm{b})$ and cation density $c_{0}(\mathrm{c}, \mathrm{d})$ on the IMVS response. Subfigures $(\mathrm{a}, \mathrm{c})$ show the Nyquist plot with corresponding imaginary component spectra depicted in $(b, d)$, demonstrating the effect of the ionic subsystem on the low-frequency response.

the high-frequency splitting. A simultaneous increase of both the electron and hole mobilities leads to a shift of both highfrequency arcs, revealing that both arcs can be attributed to a transport process. We do not attribute the two high-frequency arcs to different transport times of electrons and holes, as different mobilities $\mu_{n} \neq \mu_{p}$ generate an additional arc (corresponding to the transport of electrons and holes, respectively). Changing the ionic density in the perovskite layer severely influences the potential and hence the distribution of electrons and holes across the layer. We believe that the high-frequency splitting for equal motility $\mu_{n}=\mu_{p}$ and low ion concentration as shown in Figure $3 \mathrm{~d}$ are explicable by the nonuniform charge distribution across the perovskite layer, which leads to two distinguishable time constants. We note that the high-frequency response has been reported to be dominated by $R C$ attenuation, ${ }^{15}$ which is neglected in Figure 3. The high-frequency splitting as discussed here is therefore presumably not observable experimentally. The effect of $R C$ attenuation is discussed in the next section.

The IMVS response is characterized by two different time constants, which are linked to the electronic and ionic subsystems. The influence of parameters associated with 

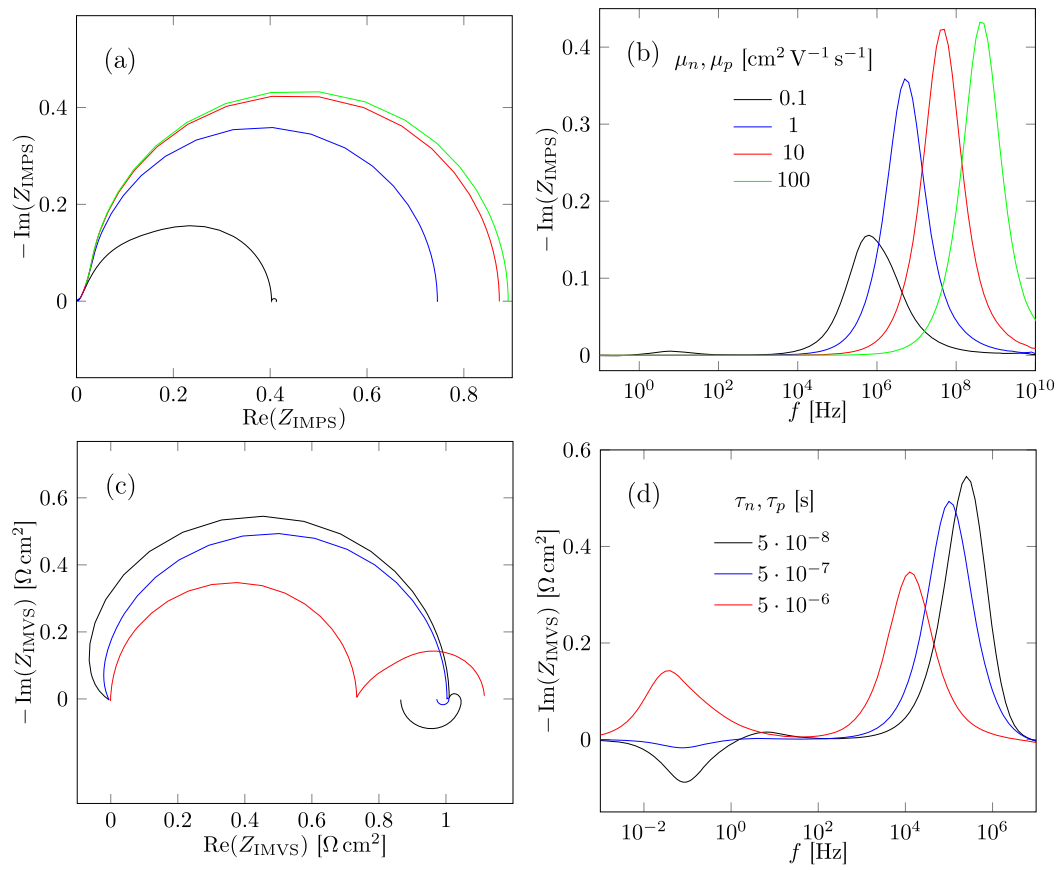

Figure 5. Simulated IMPS response (a,b) for varying mobilities $\mu_{n}, \mu_{p}$ of electrons and holes and simulated IMVS response (c,d) for varying SRH lifetimes $\tau_{n}, \tau_{p}$. Parts $(\mathrm{a}, \mathrm{c})$ show the Nyquist plot and parts $(\mathrm{b}, \mathrm{d})$ show the imaginary part of the transfer function, demonstrating the effect of transport and recombination of electronic charges on the high-frequency response. We assumed perfectly selective contacts $v_{p}^{0}=v_{n}^{d}=0$ for the simulation of IMVS.

mobile ions is shown in Figure 4. Increasing the mobility or density of cations causes a shift of the slow component to higher frequencies. The same reasoning as for the IMPS results applies here.

3.2. Analysis of the High-Frequency Response. The influence of parameters associated with the electronic subsystem on the IMPS and IMVS results is shown in Figure 5. The high-frequency time constant of the IMVS response is interpreted as carrier lifetime. ${ }^{15,52}$ For perfectly selective contacts, $v_{p}^{0}=v_{n}^{d}=0$, the high-frequency time constant can be associated with the bulk lifetime. We have neglected surface recombination in Figure 5d for clarity, as superposition of the two recombination processes makes association of the highfrequency time constant less apparent. The high-frequency time constant of IMPS results is a measure for the mean transport time of generated carriers to the respective contacts and is hence related to the mobility of electrons and holes. ${ }^{52}$ We illustrate the dependence of the high-frequency response on the charge carrier mobilities in Figure $5 \mathrm{~b}$, where $R C$ attenuation has been neglected. Note that the high-frequency IMPS response associated with charge transport is not measurable for PSCs, as it is dominated by the $R C$ time constant. ${ }^{15}$ We will address $R C$ effects later in this section.

The parameters associated with the electronic subsystem not only affect the high-frequency response but also have an impact on the low-frequency results. Vice versa, the parameters associated with the ionic subsystem may influence the highfrequency response, cf. Figure $3 \mathrm{~d}$. The additional charges generated by light perturbation modify the electric field within the perovskite layer, forcing the mobile ions to equilibrate. This ionic current again affects the transport and recombination of electronic charges via the potential. These coupling effects are in line with the models used to interpret the impedance spectra of PSCs, ${ }^{53,54}$ where the modulation of the electronic recombination, charge injection, and transport caused by ion redistribution have been discussed.

The high-frequency IMPS response has been reported to be dominated by the time constant $R C_{\text {geo }}{ }^{15,16,40}$ We show the effect of $R C$ attenuation on simulated IMPS results in Figure 6 for $C=C_{\text {geo }}=\varepsilon_{\mathrm{r}} \varepsilon_{0} / d$ and $R=1-100 \Omega \mathrm{cm}^{2}$, covering a wide range of values reported in the literature. ${ }^{15,16,55}$ For a resistance of $R=1 \Omega \mathrm{cm}^{2}$, the associated time constant $\tau_{R C}$
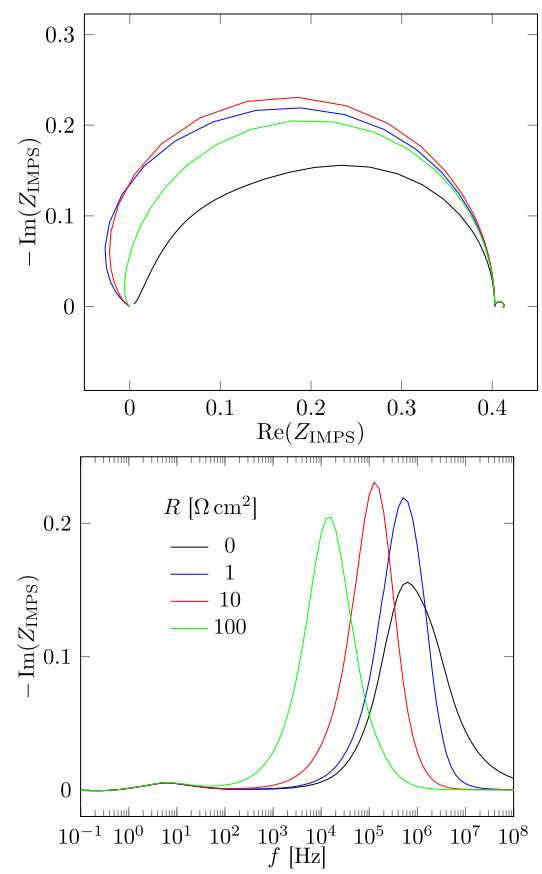

Figure 6. Simulated IMPS response for varying resistance $R$, illustrating the effect of $R C$ attenuation with $C=C_{\text {geo }}=\varepsilon_{\mathrm{r}} \varepsilon_{0} / d$. 
$\approx 1.1 \times 10^{-7} \mathrm{~s}$ is roughly in the same range as the time constant related to charge transport. For resistances of the order $R \geq 10 \Omega \mathrm{cm}^{2}$, the high-frequency response is completely dominated by $R C$ effects. We have used electron and hole mobilities of $\mu_{n}=\mu_{p}=0.1 \mathrm{~cm}^{2} \mathrm{~V}^{-1} \mathrm{~s}^{-1}$ for our simulations, which are on the lower end of the reported values. ${ }^{32,33}$ For mobilities given by $\mu_{n}=\mu_{p} \geq 1 \mathrm{~cm}^{2} \mathrm{~V}^{-1} \mathrm{~s}^{-1}$, the high-frequency response is dominated by $R C$ attenuation even for very low resistances of the order $R=1 \Omega \mathrm{cm}^{2}$. Our simulation results confirm that the high-frequency IMPS response associated with transport of electronic charges is dominated by the $R C$ time constant. Recently, it has also been shown for transient photovoltage (which is the time equivalent of IMVS) that the extracted time constant is governed by recombination at high light intensities but dominated by $R C$ effects in the limit of low light intensities. ${ }^{56}$

At high frequencies, both the IMPS and IMVS transfer functions may cross the imaginary axis, cf. Figures 5 and 6 . The negative real parts of the transfer functions at high frequencies have been discussed in refs 52 and 57. In the case of IMPS, this feature is caused by $R C$ attenuation. The negative real parts of the IMVS transfer functions have been associated with nonuniform charge generation by Halme et al. ${ }^{52}$ As we use the Lambert-Beer model for the generation of electrons and holes, we obtain a similar spiraling to the origin at high frequencies.

3.3. Simulation of IMPS and IMVS for Varied Irradiance. The dependence of the simulated IMVS response on illumination intensity is depicted in Figure 7. It is characterized by a high-frequency time constant, which is inversely proportional to the light intensity, $\tau_{\mathrm{hf}}^{\mathrm{IMVS}} \propto \Phi_{\mathrm{ph}}{ }^{-1}$, and a low-frequency time constant, which shows negligible dependence on illumination intensity. The trend of these time constants is in agreement with the measurements
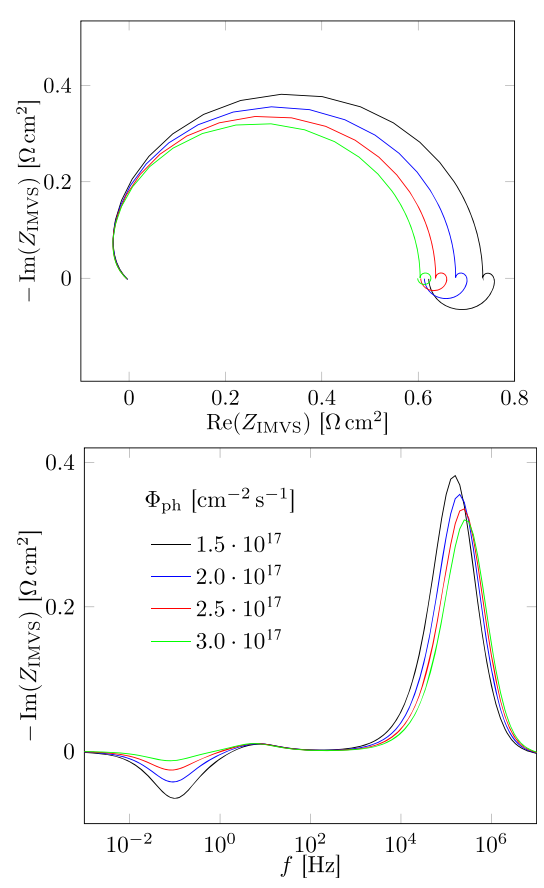

Figure 7. Simulated IMVS response for varying illumination intensity. While the high-frequency time constant is inversely proportional to the light intensity, the low-frequency time constant shows negligible dependence on the illumination intensity. reported in refs 40 and 46. Our simulation results differ qualitatively from the reported measurements as the simulated low-frequency peak appears in the first quadrant while the measurements remain exclusively in the fourth quadrant. This difference is discussed in the next section.

For low intensities, the total capacitance of the device is dominated by the geometric capacitance $C_{\text {geo }}$ and is thus independent of the irradiance. The recombination resistance $R_{\text {rec }}$ is inversely proportional to the light intensity and so is $\tau_{\mathrm{hf}}^{\mathrm{IMV}}=R_{\mathrm{rec}} C_{\mathrm{geo}}$, and the simulation results are thus consistent with the discussion given in ref 15 . For large photon fluxes $\left(\Phi_{\mathrm{ph}}>3 \times 10^{17} \mathrm{~cm}^{-2} \mathrm{~s}^{-1}\right.$ and parameters as in Table 1$)$, we obtain chemical capacitances of the order of the geometric capacitance, leading to deviations from the relation $\tau_{\mathrm{hf}}^{\mathrm{IMVS}} \propto$ $\Phi_{\mathrm{ph}}{ }^{-1}$.

For the simulation of IMPS, we set the series resistance to $R$ $=50 \Omega \mathrm{cm}^{2}$. The high-frequency response is dominated by the time constant equivalent to $f \approx 2.9 \times 10^{4} \mathrm{~Hz}$, obscuring the effect of electronic charge carrier transport. ${ }^{14,16}$ The simulated IMPS results in Figure 8 are thus characterized by a high-
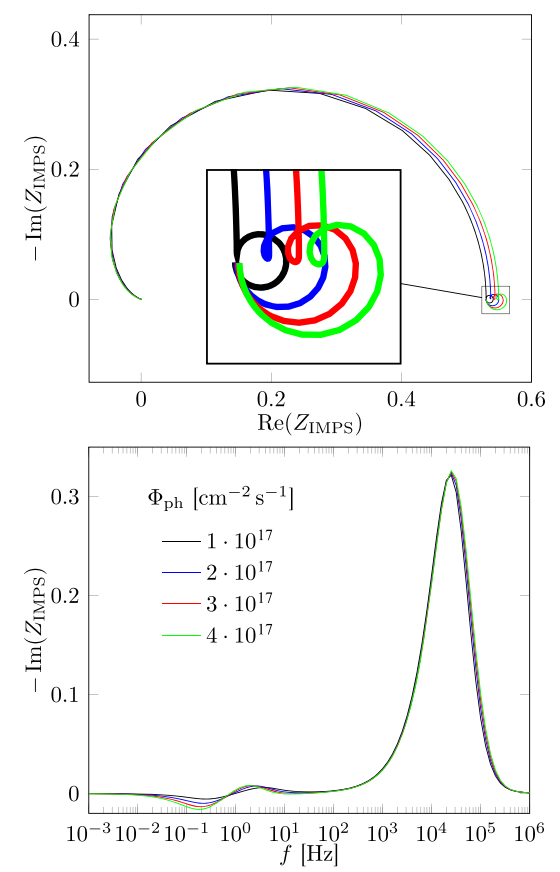

Figure 8. Simulated IMPS response for varying light intensity. We set $R=50 \Omega \mathrm{cm}^{2}, \tau_{n}=\tau_{p}=1.6 \times 10^{-6} \mathrm{~s}$, and $\mu_{n}=\mu_{p}=0.01 \mathrm{~cm}^{2} \mathrm{~V}^{-1} \mathrm{~s}^{-1}$ so that the simulation results qualitatively match the measurements reported in ref 44 .

frequency arc in the upper quadrant dominated by $R C$ attenuation and independent of illumination intensity, followed by a low-frequency arc in the lower quadrant, which is more pronounced for higher light intensities. A lowfrequency arc in the first quadrant with similar trends to light intensity has been reported in ref 44 .

3.4. Linking the Time and Frequency Domain. The FD method links the time and frequency domain, which can facilitate the interpretation of IMPS and IMVS results. The transfer into time domain has been used to reveal the origin of negative capacitance measured in the impedance spectra of organic solar cells ${ }^{21}$ and more recently in PSCs. ${ }^{54}$ We discuss the correlation of time and frequency domain exemplarily for the influence of the SRV on the IMVS response. Figure $9 a, b$ 

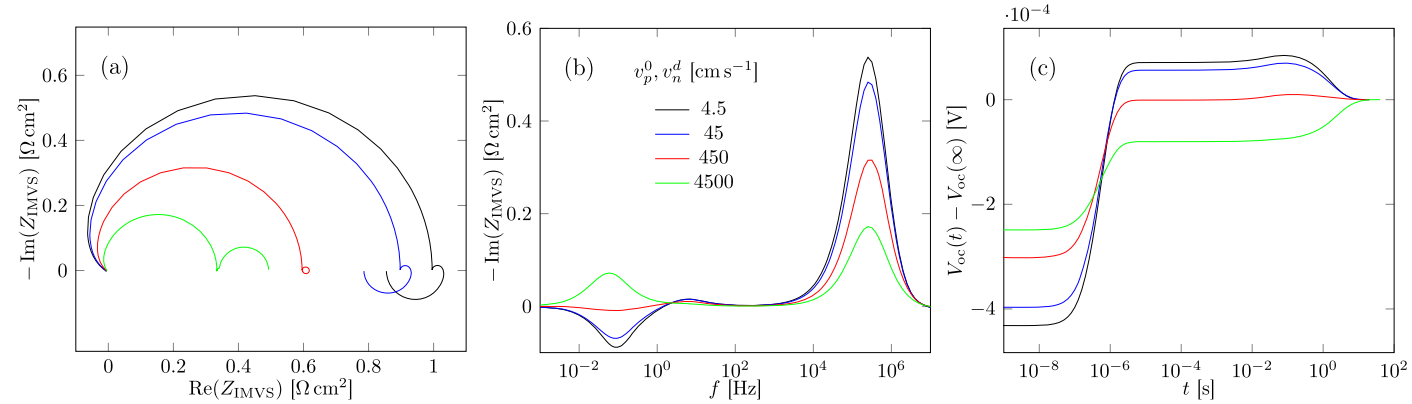

Figure 9. Simulated IMVS response (a,b) for varying SRV of the minority carrier. (c) Transient photovoltage response to a small light intensity step at $t=0$.

reveals a considerable effect of the surface recombination on the IMVS results. The transient open-circuit voltage response to a small intensity step at $t=0$ is depicted in Figure 9c, which is characterized by a first increase on the microsecond time scale followed by further relaxation at the order of seconds.

For high surface recombination, the IMVS response is exclusively in the fourth quadrant, while for reduced surface recombination, the low-frequency arc of the IMVS response crosses the real axis. This feature can be explained in the time domain by the transient behavior of the open-circuit voltage. The real and imaginary parts of the transfer function are determined by the Fourier sine and cosine transforms of the transient open-circuit voltage relative to its stationary value, $\mathrm{cf}$. eq 17 . In the low-frequency limit, we set $\cos (\omega t) \equiv 1$, which results in the integral $\int_{0}^{\infty} V(t)-V(\infty) \mathrm{d} t$. The sign of this integral reveals whether $Z_{\text {IMVs }}$ will tend to the real axis from the upper or lower half plane. For low surface recombination velocities, this integral becomes positive [the sign of the integral is easily read off (Figure 9c), considering the logarithmically plotted time axis], which results in $\operatorname{Im}\left(Z_{\text {IMVs }}\right)$ $>0$ for low frequencies.

The crossing of the real axis of the IMVS transfer function can thus be attributed to the nonmonotonic behavior of the open-circuit voltage after an intensity step. This nonmonotonicity can be explained by the ambiguous effect of ionic redistribution on the open-circuit voltage dependent on the surface recombination, yet a detailed analysis of this issue is not within the scope of this paper. For the discussion of nonmonotonic photovoltage transients of cells subjected to a change in light intensity, we refer to Calado et al. ${ }^{5}$ and Walter et al. $^{10}$

3.5. Coupling of the Electronic and Ionic Subsystems. To tackle the numerical complexity of the system $(1-4)$, a different solution strategy has been suggested by Richardson et al. The underlying assumption made therein is that the contribution of electronic charges to the potential is negligible. This results in a decoupled ionic subsystem, which has been described analytically with an expression for the potential drop across Debye layers. Electrons and holes have further been assumed to equilibrate instantaneously.

This approach reduces the demands on spatial and temporal discretization. However, it results in a "one-way coupled system", as the ionic distribution affects the electronic subsystem via the potential, but the ionic subsystem does not react to changes of electronic charges. Consequently, we name the approach chosen in the present paper "fully coupled system". We use the expression "fully coupled system" for distinction of the two strategies, establishing the fully coupled system is not an achievement of the present paper. In fact, the fully coupled system has been solved in ref 4 for a more favorable set of parameters, and it has been solved in the MIEC and LEC community before PSCs have emerged. ${ }^{11,12}$

We argue that there is no need for the aforementioned simplifications as it is indeed possible to resolve Debye layers by suitable numerical tools even for challenging sets of parameters. We emphasize the necessity to solve the fully coupled set of equations for the simulation of IMPS and IMVS, as the perturbation is induced in the electronic subsystem by light modulation. We take the view that one-way coupling is not suitable for this type of problem, as the ions would not react to the excitation. An equivalent circuit model in conjunction with one-way coupling has been suggested by Domanski et al. ${ }^{8}$ to model IMPS, yet the analytic expression derived therein admits only a single time constant.

The validity of one-way coupling has been demonstrated for current-voltage curves in refs 4 and 24, though it has subsequently also been applied for the investigation of transient effects on time scales below seconds. ${ }^{8,9}$ In order to reveal the differences between one-way and full coupling, we recalculate the transient cell response to a voltage step, which has been discussed within the one-way coupled system by O'Kane et al. ${ }^{9}$ Therein, the electronic charges equilibrate instantaneously and the potential is fully determined by the ionic distribution, leading to a cell response which is characterized by a single time constant attributed to the ionic redistribution. This is in contrast to the fully coupled system, where two time constants related to electronic charges and ions evolve.

For one-way coupling, we reproduce exactly the simulation results presented in ref 9 , which further verifies our simulation results. In contrast, we obtain considerable qualitative deviations for time scales below seconds with the fully coupled system, cf. Figure 10. The difference is caused by the redistribution of holes, which react much quicker than ions to the applied potential. This leads locally to hole concentrations exceeding ion concentrations near the anode, even though the total ion concentration within the cell is by several orders of magnitude higher than the total hole concentration. In the fully coupled system, this results in an electric field, which is not exclusively determined by the distribution of ions.

\section{CONCLUSIONS}

The IMPS and IMVS response of PSCs has been analyzed in the setting of a one-dimensional drift-diffusion model. The small signal behavior of a device exposed to light intensity perturbation has been numerically derived with $S^{3} A$ and FD of transient excitation, two standard techniques usually employed 


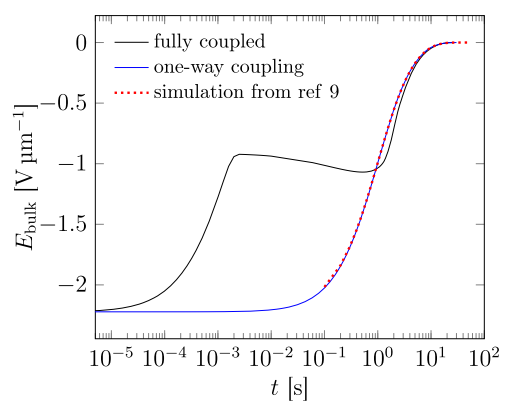

Figure 10. Simulated cell response to a voltage step at $t=0$ using oneway coupling (blue line) and the fully coupled system (black line) with a set of parameters taken from ref 9 . The dotted simulation results are reprinted with permission from ref 9-Published by The Royal Society of Chemistry.

in the context of numerical device simulation of IS. The FD method correlates the frequency and time domain, the latter of which is often easier to interpret. The necessity to consider full coupling of the electronic and ionic subsystems has been discussed.

Simulated IMPS and IMVS responses exhibit two clearly distinguishable time constants. The high-frequency response is associated with the transport and recombination of electronic charges, while the low-frequency response is attributed to the redistribution of mobile ions. The effect of ion transport on the IMPS and IMVS response predicted by our model has been thoroughly analyzed. Exemplarily for varied illumination intensity, a parameter study has shown that the simulation results are in good qualitative agreement with measurements reported in the literature.

In summary, we have presented a model which is capable of explaining the qualitative IMPS and IMVS behavior of PSCs. The simplicity of the model makes it particularly suitable for the interpretation of IMPS and IMVS results and to associate the high- and low-frequency response to the underlying physical processes. It builds further evidence for the plethora of novel dynamic effects exhibited by PSCs which are explicable by ion movement. To achieve quantitative fitting for parameter extraction, a more detailed model considering the charge transport in the ETL and HTL would be necessary.

\section{AUTHOR INFORMATION}

\section{Corresponding Author}

*E-mail: david.bernhardsgruetter@zhaw.ch.

\section{ORCID}

David Bernhardsgrütter: 0000-0001-5033-5974

Notes

The authors declare no competing financial interest.

\section{ACKNOWLEDGMENTS}

Financial funding from the Swiss National Science Foundation, project no 407040-153952, is gratefully appreciated.

\section{REFERENCES}

(1) Kojima, A.; Teshima, K.; Shirai, Y.; Miyasaka, T. Organometal Halide Perovskites as Visible-Light Sensitizers for Photovoltaic Cells. J. Am. Chem. Soc. 2009, 131, 6050-6051.

(2) National Renewable Energy Laboratory. Best Research-Cell Efficiency Chart. 2019. https://www.nrel.gov/pv/assets/pdfs/bestresearch-cell-efficiencies.20190923.pdf (accessed Oct 28, 2019).
(3) van Reenen, S.; Kemerink, M.; Snaith, H. J. Modeling Anomalous Hysteresis in Perovskite Solar Cells. J. Phys. Chem. Lett. 2015, 6, 3808-3814.

(4) Richardson, G.; O’Kane, S. E. J.; Niemann, R. G.; Peltola, T. A.; Foster, J. M.; Cameron, P. J.; Walker, A. B. Can Slow-Moving Ions Explain Hysteresis in the Current-Voltage Curves of Perovskite Solar Cells? Energy Environ. Sci. 2016, 9, 1476-1485.

(5) Calado, P.; Telford, A. M.; Bryant, D.; Li, X.; Nelson, J.; O'Regan, B. C.; Barnes, P. R. Evidence for Ion Migration in Hybrid Perovskite Solar Cells with Minimal Hysteresis. Nat. Commun. 2016, $7,13831$.

(6) Jacobs, D. A.; Wu, Y.; Shen, H.; Barugkin, C.; Beck, F. J.; White, T. P.; Weber, K.; Catchpole, K. R. Hysteresis Phenomena in Perovskite Solar Cells: The Many and Varied Effects of Ionic Accumulation. Phys. Chem. Chem. Phys. 2017, 19, 3094-3103.

(7) Neukom, M. T.; Züfle, S.; Knapp, E.; Makha, M.; Hany, R.; Ruhstaller, B. Why Perovskite Solar Cells with High Efficiency Show Small IV-Curve Hysteresis. Sol. Energy Mater. Sol. Cells 2017, 169, $159-166$.

(8) Domanski, K.; Roose, B.; Matsui, T.; Saliba, M.; Turren-Cruz, S.H.; Correa-Baena, J.-P.; Carmona, C. R.; Richardson, G.; Foster, J. M.; De Angelis, F.; et al. Migration of Cations Induces Reversible Performance Losses over Day/Night Cycling in Perovskite Solar Cells. Energy Environ. Sci. 2017, 10, 604-613.

(9) O'Kane, S. E. J.; Richardson, G.; Pockett, A.; Niemann, R. G.; Cave, J. M.; Sakai, N.; Eperon, G. E.; Snaith, H. J.; Foster, J. M.; Cameron, P. J.; et al. Measurement and Modelling of Dark Current Decay Transients in Perovskite Solar Cells. J. Mater. Chem. C 2017, 5, 452-462.

(10) Walter, D.; Fell, A.; Wu, Y.; Duong, T.; Barugkin, C.; Wu, N.; White, T.; Weber, K. Transient Photovoltage in Perovskite Solar Cells: Interaction of Trap-Mediated Recombination and Migration of Multiple Ionic Species. J. Phys. Chem. C 2018, 122, 11270-11281.

(11) Mills, T. J.; Lonergan, M. C. Charge Injection and Transport in Low-Mobility Mixed Ionic/Electronic Conducting Systems: Regimes of Behavior and Limiting Cases. Phys. Rev. B: Condens. Matter Mater. Phys. 2012, 85, 035203.

(12) Buda, M.; Kalyuzhny, G.; Bard, A. J. Thin-Film Solid-State Electroluminescent Devices Based On Tris(2,2'-bipyridine)ruthenium(II) Complexes. J. Am. Chem. Soc. 2002, 124, 6090-6098.

(13) Neukom, M. T.; Schiller, A.; Züfle, S.; Knapp, E.; Ávila, J.; Pérez-del-Rey, D.; Dreessen, C.; Zanoni, K. P. S.; Sessolo, M.; Bolink, H. J.; et al. Consistent Device Simulation Model Describing Perovskite Solar Cells in Steady-State, Transient, and Frequency Domain. ACS Appl. Mater. Interfaces 2019, 11, 23320-23328.

(14) Guillén, E.; Ramos, F. J.; Anta, J. A.; Ahmad, S. Elucidating Transport-Recombination Mechanisms in Perovskite Solar Cells by Small-Perturbation Techniques. J. Phys. Chem. C 2014, 118, 2291322922.

(15) Pockett, A.; Eperon, G. E.; Peltola, T.; Snaith, H. J.; Walker, A.; Peter, L. M.; Cameron, P. J. Characterization of Planar Lead Halide Perovskite Solar Cells by Impedance Spectroscopy, Open-Circuit Photovoltage Decay, and Intensity-Modulated Photovoltage/Photocurrent Spectroscopy. J. Phys. Chem. C 2015, 119, 3456-3465.

(16) Ravishankar, S.; Aranda, C.; Sanchez, S.; Bisquert, J.; Saliba, M.; Garcia-Belmonte, G. Perovskite Solar Cell Modeling Using Light- and Voltage-Modulated Techniques. J. Phys. Chem. C 2019, 123, 64446449.

(17) Tress, W. Organic Solar Cells: Theory, Experiment, and Device Simulation; Springer: Cham Heidelberg New York Dordrecht London, 2014.

(18) Dloczik, L.; Ileperuma, O.; Lauermann, I.; Peter, L. M.; Ponomarev, E. A.; Redmond, G.; Shaw, N. J.; Uhlendorf, I. Dynamic Response of Dye-Sensitized Nanocrystalline Solar Cells: Characterization by Intensity-Modulated Photocurrent Spectroscopy. J. Phys. Chem. B 1997, 101, 10281-10289.

(19) Laux, S. E. Techniques for Small-Signal Analysis of Semiconductor Devices. IEEE Trans. Comput. Aided Des. Integr Circuits Syst. 1985, 4, 472-481. 
(20) Ershov, M.; Liu, H. C.; Li, L.; Buchanan, M.; Wasilewski, Z. R.; Jonscher, A. K. Negative Capacitance Effect in Semiconductor Devices. IEEE Trans. Electron Devices 1998, 45, 2196-2206.

(21) Knapp, E.; Ruhstaller, B. Analysis of Negative Capacitance and Self-Heating in Organic Semiconductor Devices. J. Appl. Phys. 2015, 117, 135501.

(22) Eames, C.; Frost, J. M.; Barnes, P. R. F.; O’Regan, B. C.; Walsh, A.; Islam, M. S. Ionic Transport in Hybrid Lead Iodide Perovskite Solar Cells. Nat. Commun. 2015, 6, 7497.

(23) Yang, Y.; Yang, M.; Moore, D. T.; Yan, Y.; Miller, E. M.; Zhu, K.; Beard, M. C. Top and Bottom Surfaces Limit Carrier Lifetime in Lead Iodide Perovskite Films. Nat. Energy 2017, 2, 16207.

(24) Courtier, N. E.; Foster, J. M.; O’Kane, S. E. J.; Walker, A. B.; Richardson, G. Systematic Derivation of a Surface Polarisation Model for Planar Perovskite Solar Cells. Eur. J. Appl. Math. 2019, 30, 427.

(25) Anusca, I.; Balčiūnas, S.; Gemeiner, P.; Svirskas, S.; Sanlialp, M.; Lackner, G.; Fettkenhauer, C.; Belovickis, J.; Samulionis, V.; Ivanov, M.; et al. Dielectric Response: Answer to Many Questions in the Methylammonium Lead Halide Solar Cell Absorbers. Adv. Energy Mater. 2017, 7, 1700600.

(26) Zhou, Y.; Long, G. Low Density of Conduction and Valence Band States Contribute to the High Open-Circuit Voltage in Perovskite Solar Cells. J. Phys. Chem. C 2017, 121, 1455-1462.

(27) Walsh, A.; Scanlon, D. O.; Chen, S.; Gong, X. G.; Wei, S.-H. Self-Regulation Mechanism for Charged Point Defects in Hybrid Halide Perovskites. Angew. Chem., Int. Ed. 2015, 54, 1791-1794.

(28) Johnston, M. B.; Herz, L. M. Hybrid Perovskites for Photovoltaics: Charge-Carrier Recombination, Diffusion, and Radiative Efficiencies. Acc. Chem. Res. 2016, 49, 146-154.

(29) Tress, W. Perovskite Solar Cells on the Way to Their Radiative Efficiency Limit-Insights Into a Success Story of High Open-Circuit Voltage and Low Recombination. Adv. Energy Mater. 2017, 7, 1602358.

(30) Löper, P.; Stuckelberger, M.; Niesen, B.; Werner, J.; Filipič, M.; Moon, S.-J.; Yum, J.-H.; Topič, M.; De Wolf, S.; Ballif, C. Complex Refractive Index Spectra of CH3NH3PbI3 Perovskite Thin Films Determined by Spectroscopic Ellipsometry and Spectrophotometry. J. Phys. Chem. Lett. 2015, 6, 66-71.

(31) Tao, S.; Schmidt, I.; Brocks, G.; Jiang, J.; Tranca, I.; Meerholz, K.; Olthof, S. Absolute Energy Level Positions in Tin- and Lead-Based Halide Perovskites. Nat. Commun. 2019, 10, 2560.

(32) Maynard, B.; Long, Q.; Schiff, E. A.; Yang, M.; Zhu, K.; Kottokkaran, R.; Abbas, H.; Dalal, V. L. Electron and Hole Drift Mobility Measurements on Methylammonium Lead Iodide Perovskite Solar Cells. Appl. Phys. Lett. 2016, 108, 173505.

(33) Herz, L. M. Charge-Carrier Mobilities in Metal Halide Perovskites: Fundamental Mechanisms and Limits. ACS Energy Lett. 2017, 2, 1539-1548.

(34) Shi, J.; Li, Y.; Li, Y.; Li, D.; Luo, Y.; Wu, H.; Meng, Q. From Ultrafast to Ultraslow: Charge-Carrier Dynamics of Perovskite Solar Cells. Joule 2018, 2, 879-901.

(35) Correa-Baena, J.-P.; Anaya, M.; Lozano, G.; Tress, W.; Domanski, K.; Saliba, M.; Matsui, T.; Jacobsson, T. J.; Calvo, M. E.; Abate, A.; et al. Unbroken Perovskite: Interplay of Morphology, Electro-Optical Properties, and Ionic Movement. Adv. Mater. 2016, 28, 5031-5037.

(36) Contreras, L.; Idígoras, J.; Todinova, A.; Salado, M.; Kazim, S.; Ahmad, S.; Anta, J. A. Specific Cation Interactions as the Cause of Slow Dynamics and Hysteresis in Dye and Perovskite Solar Cells: a Small-Perturbation Study. Phys. Chem. Chem. Phys. 2016, 18, 3103331042.

(37) Seo, J.-Y.; Matsui, T.; Luo, J.; Correa-Baena, J.-P.; Giordano, F.; Saliba, M.; Schenk, K.; Ummadisingu, A.; Domanski, K.; Hadadian, M.; et al. Ionic Liquid Control Crystal Growth to Enhance Planar Perovskite Solar Cells Efficiency. Adv. Energy Mater. 2016, 6, 1600767.

(38) Roose, B.; Ummadisingu, A.; Correa-Baena, J.-P.; Saliba, M.; Hagfeldt, A.; Graetzel, M.; Steiner, U.; Abate, A. Spontaneous Crystal
Coalescence Enables Highly Efficient Perovskite Solar Cells. Nano Energy 2017, 39, 24-29.

(39) Saliba, M.; Matsui, T.; Domanski, K.; Seo, J.-Y.; Ummadisingu, A.; Zakeeruddin, S. M.; Correa-Baena, J.-P.; Tress, W. R.; Abate, A.; Hagfeldt, A.; et al. Incorporation of Rubidium Cations into Perovskite Solar Cells Improves Photovoltaic Performance. Science 2016, 354, 206-209.

(40) Prochowicz, D.; Yadav, P.; Saliba, M.; Saski, M.; Zakeeruddin, S. M.; Lewiński, J.; Grätzel, M. Reduction in the Interfacial Trap Density of Mechanochemically Synthesized MAPbI3. ACS Appl. Mater. Interfaces 2017, 9, 28418-28425.

(41) Albadri, A.; Yadav, P.; Alotaibi, M.; Arora, N.; Alyamani, A.; Albrithen, H.; Dar, M. I.; Zakeeruddin, S. M.; Grätzel, M. Unraveling the Impact of Rubidium Incorporation on the Transport-Recombination Mechanisms in Highly Efficient Perovskite Solar Cells by Small-Perturbation Techniques. J. Phys. Chem. C 2017, 121, 2490324908.

(42) Yadav, P.; Alotaibi, M. H.; Arora, N.; Dar, M. I.; Zakeeruddin, S. M.; Grätzel, M. Influence of the Nature of A Cation on Dynamics of Charge Transfer Processes in Perovskite Solar Cells. Adv. Funct. Mater. 2017, 28, 1706073

(43) Turren-Cruz, S.-H.; Saliba, M.; Mayer, M. T.; JuárezSantiesteban, H.; Mathew, X.; Nienhaus, L.; Tress, W.; Erodici, M. P.; Sher, M.-J.; Bawendi, M. G.; et al. Enhanced Charge Carrier Mobility and Lifetime Suppress Hysteresis and Improve Efficiency in Planar Perovskite Solar Cells. Energy Environ. Sci. 2018, 11, 78-86.

(44) Ravishankar, S.; Aranda, C.; Boix, P. P.; Anta, J. A.; Bisquert, J.; Garcia-Belmonte, G. Effects of Frequency Dependence of the External Quantum Efficiency of Perovskite Solar Cells. J. Phys. Chem. Lett. 2018, 9, 3099-3104.

(45) Pockett, A.; Eperon, G. E.; Sakai, N.; Snaith, H. J.; Peter, L. M.; Cameron, P. J. Microseconds, Milliseconds and Seconds: Deconvoluting the Dynamic Behaviour of Planar Perovskite Solar Cells. Phys. Chem. Chem. Phys. 2017, 19, 5959-5970.

(46) Chen, X.; Shirai, Y.; Yanagida, M.; Miyano, K. Photocarrier Dynamics in Perovskite-Based Solar Cells Revealed by IntensityModulated Photovoltage Spectroscopy. Phys. Chem. Chem. Phys. 2018, 20, 17918-17926.

(47) Cai, F.; Yan, Y.; Yao, J.; Wang, P.; Wang, H.; Gurney, R. S.; Liu, D.; Wang, T. Ionic Additive Engineering Toward High-Efficiency Perovskite Solar Cells with Reduced Grain Boundaries and Trap Density. Adv. Funct. Mater. 2018, 28, 1801985.

(48) Shao, Y.; Fang, Y.; Li, T.; Wang, Q.; Dong, Q.; Deng, Y.; Yuan, Y.; Wei, H.; Wang, M.; Gruverman, A.; et al. Grain boundary dominated ion migration in polycrystalline organic-inorganic halide perovskite films. Energy Environ. Sci. 2016, 9, 1752-1759.

(49) Yang, B.; Brown, C. C.; Huang, J.; Collins, L.; Sang, X.; Unocic, R. R.; Jesse, S.; Kalinin, S. V.; Belianinov, A.; Jakowski, J.; et al. Enhancing Ion Migration in Grain Boundaries of Hybrid OrganicInorganic Perovskites by Chlorine. Adv. Funct. Mater. 2017, 27, 1700749.

(50) Yun, J. S.; Seidel, J.; Kim, J.; Soufiani, A. M.; Huang, S.; Lau, J.; Jeon, N. J.; Seok, S. I.; Green, M. A.; Ho-Baillie, A. Critical Role of Grain Boundaries for Ion Migration in Formamidinium and Methylammonium Lead Halide Perovskite Solar Cells. Adv. Energy Mater. 2016, 6, 1600330.

(51) Giordano, F.; Abate, A.; Correa Baena, J. P.; Saliba, M.; Matsui, T.; Im, S. H.; Zakeeruddin, S. M.; Nazeeruddin, M. K.; Hagfeldt, A.; Graetzel, M. Enhanced Electronic Properties in Mesoporous TiO2 via Lithium Doping for High-Efficiency Perovskite Solar Cells. Nat. Commun. 2016, 7, 10379.

(52) Halme, J.; Miettunen, K.; Lund, P. Effect of Nonuniform Generation and Inefficient Collection of Electrons on the Dynamic Photocurrent and Photovoltage Response of Nanostructured Photoelectrodes. J. Phys. Chem. C 2008, 112, 20491-20504.

(53) Moia, D.; Gelmetti, I.; Calado, P.; Fisher, W.; Stringer, M.; Game, O.; Hu, Y.; Docampo, P.; Lidzey, D.; Palomares, E.; et al. Ionic-to-Electronic Current Amplification in Hybrid Perovskite Solar Cells: Ionically Gated Transistor-Interface Circuit Model Explains 
Hysteresis and Impedance of Mixed Conducting Devices. Energy Environ. Sci. 2019, 12, 1296-1308.

(54) Ebadi, F.; Taghavinia, N.; Mohammadpour, R.; Hagfeldt, A.; Tress, W. Origin of Apparent Light-Enhanced and Negative Capacitance in Perovskite Solar Cells. Nat. Commun. 2019, 10, 1574.

(55) Mundhaas, N.; Yu, Z. J.; Bush, K. A.; Wang, H. P.; Häusele, J.; Kavadiya, S.; McGehee, M. D.; Holman, Z. C. Series Resistance Measurements of Perovskite Solar Cells Using $\mathrm{J}_{\mathrm{sc}}-\mathrm{V}_{\mathrm{oc}}$ Measurements. Sol. RRL 2019, 3, 1800378.

(56) Sandberg, O. J.; Tvingstedt, K.; Meredith, P.; Armin, A. Theoretical Perspective on Transient Photovoltage and Charge Extraction Techniques. J. Phys. Chem. C 2019, 123, 14261-14271.

(57) Peter, L. M.; Ponomarev, E. A.; Franco, G.; Shaw, N. J. Aspects of the Photoelectrochemistry of Nanocrystalline Systems. Electrochim. Acta 1999, 45, 549-560. 